\title{
COMPUTATIONAL MODELING OF STEEL COLUMNS SUBJECTED TO EXPERIMENTALLY SIMULATED BLASTS
}

\author{
L.K. STEWART \\ School of Civil and Environmental Engineering, Georgia Institute of Technology, USA.
}

\begin{abstract}
The development of predictive tools, such as finite element models, to calculate the response of structures subjected to vehicle-borne explosive loads has become increasingly important for the engineering and defence communities. Typically, the development of such methodologies is driven by conclusions that have been obtained via field tests; however, collecting data throughout such experiments can be problematic due to the harsh testing environment. Utilizing the University of California, San Diego Blast simulator, which can simulate explosive loads in a controlled laboratory setting, a series of experiments were conducted to investigate the performance of steel columns subjected to vehicle-borne threats and a computational model was created using the qualitative and quantitative findings from the experiments.

This paper describes, in detail, the development and calibration of the finite element model, initially discussed in, created from 17 blast simulator experiments that were validated against field tests. The finite element analysis was performed with LS-DYNA, a three dimensional, explicit, Lagrangian finite element code that uses a central difference time integration method from Livermore Software Technology Corporation. The model incorporated constitutive models to represent material behaviors of interest, specifically those with strain rate effects. Loading of the column was modeled using a previously calibrated low-density foam model and smooth particle hydrodynamic elements, where appropriate.

Keywords: Blast, columns, finite element, LS-DYNA, simulator, steel.
\end{abstract}

\section{INTRODUCTION}

The blast simulator facility [1] is located at the Robert and Natalie Englekirk Structural Engineering Center (ESEC), part of the Charles Lee Powell Structural Research Laboratories of the Jacobs School of Engineering at University of California, San Diego (UCSD). The UCSD blast simulator is a one of a kind blast mitigation and impact characterization device that emulates conventional and non-conventional explosive events without the use of explosive materials and without a fireball. The energy deposition on the target (load duration) is adjustable to less than $1 \mathrm{~ms}$ from outset of specimen loading, which is accomplished with ultra-fast, computer controlled hydraulic actuators with a combined hydraulic/high pressure nitrogen energy source called blast generators (BGs). The actuators are used conjunction with appropriate loading media, which attached to the variable masses assist in the appropriate loading conditions for various blast loads. These impulsive loads can range up to 8000 psi-ms.

Using this unique system, structural steel column experiments were conducted to produce both qualitative and quantitative data. The experimental findings were used to develop and calibrate computational models in LS-DYNA. These computational models ultimately were used as predictive tools to calculate the response of steel columns subjected to blast loads.

\section{UCSD BLAST SIMULATOR}

The blast simulator utilizes custom impacting modules that are accelerated to a prescribed velocity with an array of BGs as shown in Fig. 1. Currently the UCSD facility has six BGs, four of the earlier Type I model, named BG25, and two of the newer Type II model, called BG50. 

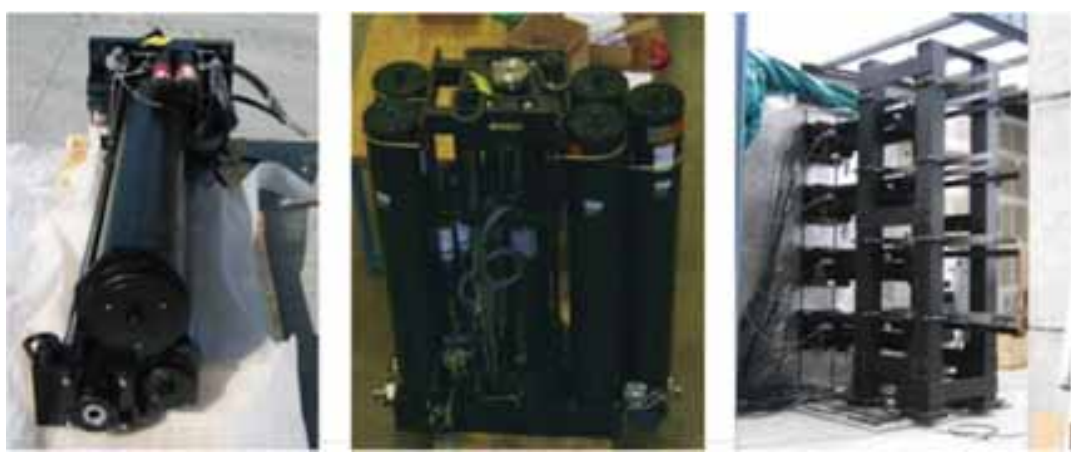

Figure 1: Blast Generator 25 (left) and Blast Generator 50 (center) and support frame (right).

The BGs consist of a hydraulic actuator, control valves, accumulators and transducers. Initially, nitrogen is compressed in the pressure accumulator along with high-pressured oil. A servocontrolled high-flow valve controls the oil flow into the actuator. Once the valve has been opened at the desired rate and amount, the oil forces the piston rod/impacting mass assembly to drive outward toward the specimen. A smaller servo-controlled valve controls the outflow of the oil and thus is able to retract the actuator after impact, thus controlling the impact duration. The force required to retract the actuator is supplied by pressurized nitrogen gas in a deceleration chamber, which is specifically calibrated before each test.

The impacting module, which consists of a steel or aluminum mass, a thin aluminum backing plate and a non-linear, urethane material called a programmer, which is used to transfer the energy and momentum of the module to the specimen. The programmer's geometric and material properties help tailor the duration and magnitude of the pressure, and thus the impulse to be representative of a blast-like pulse. The impacting modules are supported with guiderails that align the modules with the specimen until impact. The rails are supported on a large frame system, which can be adjusted for various configurations as shown in Fig. 1.

For a given structural test with varying configurations and geometry, the BGs are mounted to a fixed reaction wall, which is attached to a base isolated concrete slab. A moveable reaction wall is also attached to the slab and is used to mount specimens and fixtures.

\section{BLAST SIMULATOR EXPERIMENTS}

A total of 17 experiments were conducted on two series of columns [2,3]. The first series included columns loaded in the strong axis direction (flange impact) and the second series included columns in the weak axis direction (web impact).

\subsection{Strong axis loading experiments}

Three uniform velocity and eight variable velocity tests were conducted on five strong axis column specimens. The variable velocity tests were conducted to simulate a near field $(Z<2.0)$ vehicle-borne threat for which the impulses at the base of the column are significantly larger than those at the top of the column. The experiments were conducted on two $\mathrm{W}$ $10 \times 49$ column specimens and three W $14 \times 132$ specimens.

The test setup is illustrated in Fig. 2 with elevations of the test from the top and side. The strong axis columns were loaded with four BGs distributed over the height of the column. 

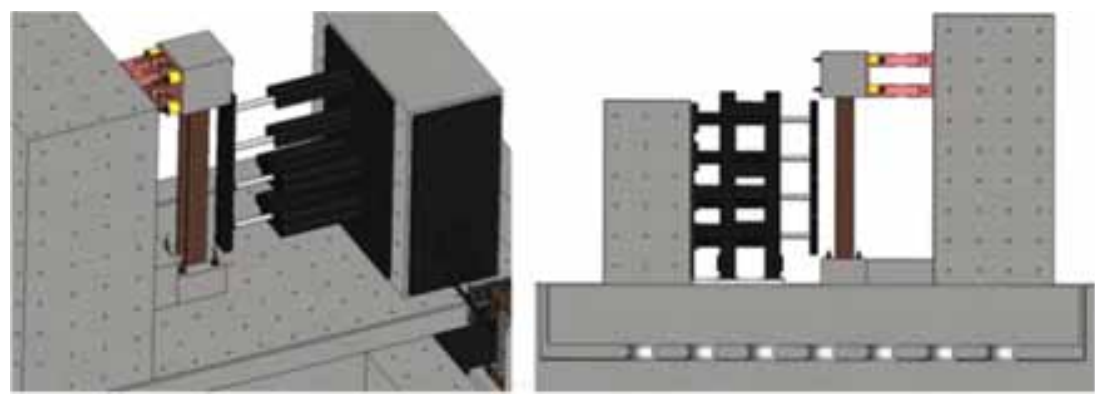

Figure 2: Experimental setup.

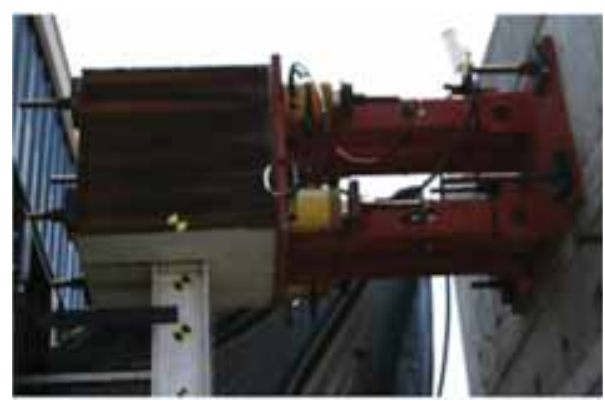

Figure 3: Link system for upper boundary condition.

For the uniform tests, four BG25s were used and for the variable velocity tests, two BG25s and two BG50s were used.

The boundary conditions for the tests were initially selected for three reasons: they were similar to the actual behavior of a building column subjected to blast loads, they were similar to conditions applied during live explosive field tests to which data and behavior could be compared, and they were thought to be simple enough for ease of modeling. The connection at the base of the column was restrained in all directions simulating a fixed condition. It was post-tensioned to the reaction floor with four rods. A concrete spacer block transferred the shear from the specimen footing to the reaction wall. The header was attached to a link system, shown in Fig. 3, which was designed to allow the column to move vertically while providing lateral and moment restraint. The link system was post-tensioned to the load stub and reaction wall. Four hollow hydraulic jacks tensioned the load stub header to the link with a 100,000 lb load and were left in place during the test.

\subsection{Weak axis loading experiments}

One uniform velocity and five variable velocity tests were conducted on six weak axis column specimens. The experiments were conducted on three W $10 \times 49$ column specimens and three W $14 \times 132$ specimens. The specimens and overall test setup used for the weak axis test series were identical to those for the strong axis tests described in Section 3.1 with the exception of the column being rotated 90 degrees. 

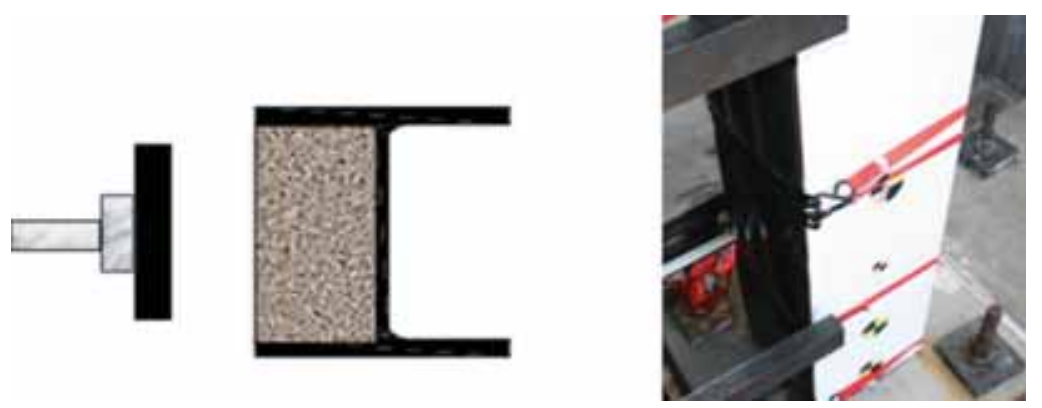

Figure 4: Loading medium bladders filled with sand or water installed into inner column area.

In order to sufficiently load the weak axis, a pressure load must be imparted on both the column web and along the insides of both column flanges. This cannot be done using a flat programmer on the web alone as was done in the previous tests. Research by Huson et al. [4] demonstrated the use of water-filled bladders as a loading medium to transfer the pressure and impulse to all inner surfaces of the column. Custom-made bladders were produced, filled with a loading medium and installed in the inside of the column, shown in Fig. 4.

Both sand and water were used as a loading medium in these experiments in order to achieve a range of responses since they behave differently under impact. The bladders were loaded with impacting modules, which varied in dimension for each of the two size specimens and test type.

\section{COMPUTATIONAL MODEL}

A finite element model for the steel columns experiments was developed for use as a tool for blast response prediction. The finite element analysis was preformed with LS-DYNA [5], a three dimensional, explicit, Lagrangian finite element code that uses a central difference time integration method. LS-DYNA is a general purpose, transient, dynamic finite element program that is often used in problems with blast and impact loading. It is able to utilize state of the art constitutive models to represent material behaviors of interest, specifically steel with strain rate effects and concrete.

\subsection{Strong axis loading model}

The strong axis experiments were modeled and are described in the following sections. The model was calibrated with the data from the simulator tests and comparisons are given in Section 5.1.

\subsubsection{Column mesh development}

Fully integrated shell elements with six integration points through the thickness were used to model the steel column. Shell elements were selected for the steel because, not only are they computationally accurate, but they also provide for a faster processing time, which is critical for developing the fast running predictive tools. The shell elements used were $0.5 \times 0.5$ in and were given the corresponding thicknesses to the column dimensions, which ranged from 0.5 to $2.5 \mathrm{in}$. The shell elements from the column flanges share nodes with the column web at the appropriate connection point. Figure 5 shows the W $14 \times 132$ steel column constructed with shell elements. 

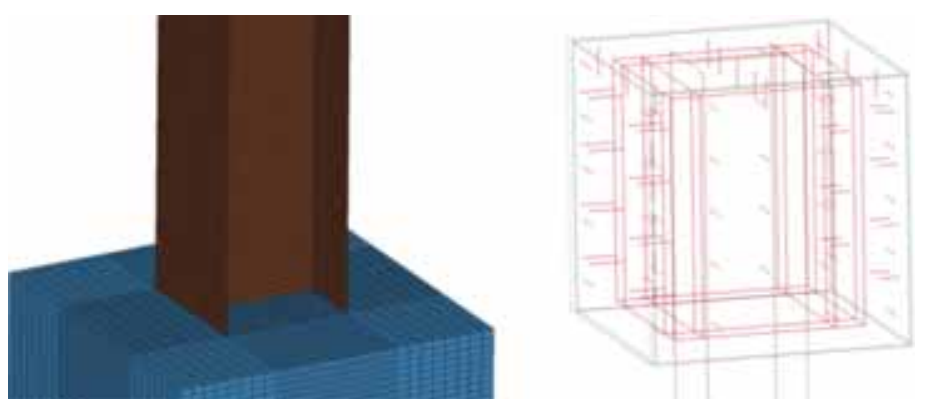

Figure 5: Column and footer mesh (left) and reinforcing steel (right).

The model for the strong axis steel column tests used three-dimensional solid elements to represent the concrete header, concrete footer, steel casing and link system. These elements were eight node brick elements with single point integration. The elements varied in size throughout the mesh. The largest element being one is located in the concrete header and footer away from the steel column. Figure 5 also shows solid elements used to model the concrete footer, which included the steel casing.

Hughes Liu beam elements with cross sectional area equal to that of the steel were used for modeling steel reinforcement and Nelson Studs in the concrete header and footer and are also shown in Fig. 5 (right). The nodes of the reinforcement were consistent with those in the solid concrete mesh.

\subsubsection{Boundary conditions}

The boundary conditions on the concrete footer were prescribed such that the nodes on the base and the four sides were restrained in all directions, which simulated the fixed condition. Correctly modeling the boundary conditions on the header was crucial in developing a calibrated model. Initially, it was assumed that the system remained fixed in the rotation and was only allowed to translate vertically and could be modeled using simple nodal restraints; however, at the higher-level velocity tests, these boundary conditions were not fully met and the system began to act more like a pin connection than a fixed connection as the velocities increased.

Three main situations were identified that contributed to the boundary conditions not being consistent for all tests. The first cause that was identified was that the hydraulic jacks could not hold the header to the link system and a gap, and therefore rotation, occurred. An example of this was captured by the high-speed video and is shown in Fig. 6.

The second scenario that contributed to the boundary condition error was the fact that, in some tests, the link system as a whole pulled off from the reaction wall. The rods that connected the link to the wall were not sufficient in fully restraining the link. This scenario was also evident in the high-speed video footage.

The last cause that was identified was that, over time, the system became loose due to opening of holes and other issues. This allowed the pins to rotate and translate within the holes and the whole system to rotate. An example of this rotation is shown in Fig. 7. The left photo was captured before impact, while the right photo was captured during the experiment.

To incorporate these factors, the entire link system was modeled as shown in Fig. 8 and connected to a steel backing plate. The backing plate has a fixed nodal restraint on the back 


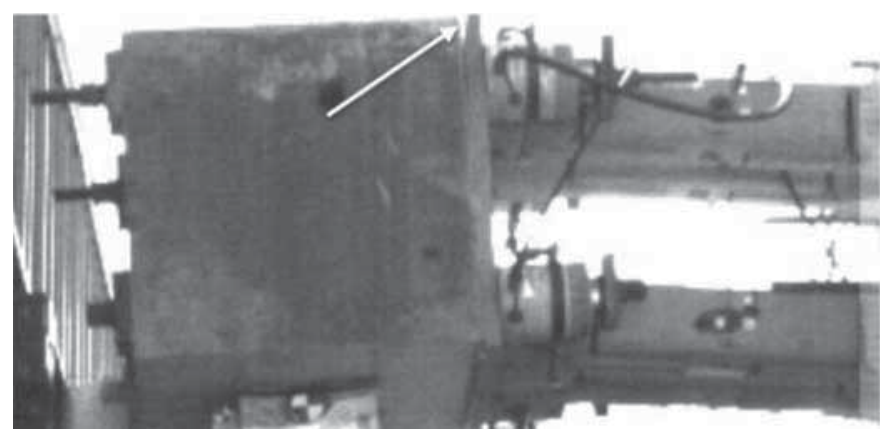

Figure 6: Header pulling away from link system.

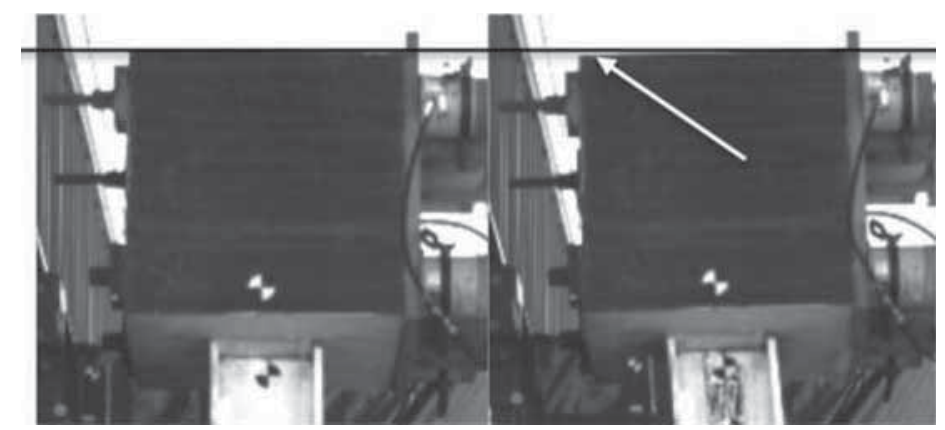

Figure 7: Rotation of loose system.
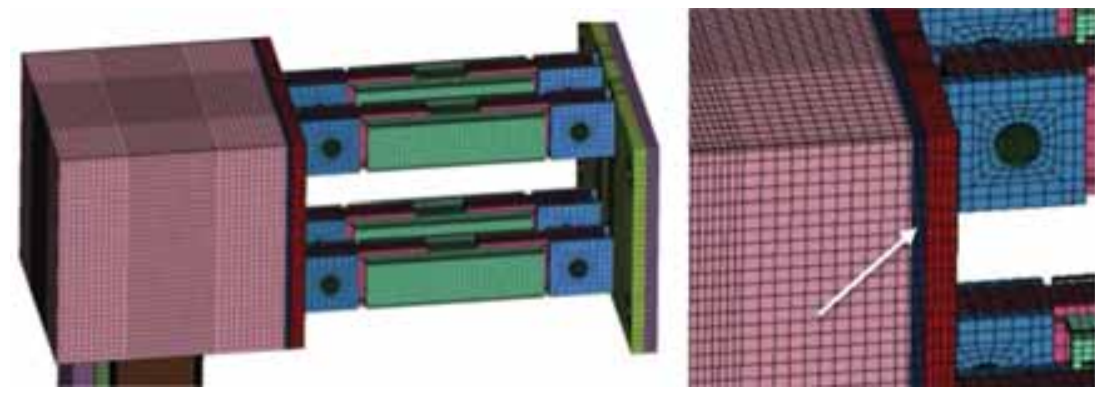

Figure 8: Link system mesh (left) and rubber connection elements (right).

of one side and was merged to the tabs, which hold the pins on the other. There is a steel tube section connecting the two sets of pins. For both sets of pins, an *AUTOMATIC_SURFACE-_TO_SURFACE contact was placed between the pin and the surrounding parts to allow rotation.

To correctly model the extra rotation tor higher velocity tests, a layer of calibrated rubber elements was placed between the link and the column header and is shown in Fig. 8. The rubber layer allowed for the link to be stiffer at small displacements as seen in the lower velocity impacts, while displacing more for the higher velocity impacts as seen in the tests. 
The rubber was four elements thick and was merged to the column and a *TIED_SURFACE_ TO_SURFACE contact was used to connect the rubber to the link, which did not share many common nodes. The material description for the rubber is discussed in Section 4.1.4.

\subsubsection{Loading}

Loads were applied to the column by modeling the impacting unit, which included the programmer, aluminum backing plate, impaction mass, rod and collar with an initial velocity. It was found that for these tests, the pyramid system on the face of the programmer did not greatly affect the results, but greatly slowed run times and, therefore, was omitted. The rod was not fully modeled, but the density of the material changed, including the full mass of the rod, as shown in Fig. 9.

\subsubsection{Material models}

To model the steel material *MAT_PIECEW1SE_LINEAR_PLASTICITY was used. This model allows for the input of arbitrary stress versus strain relations for various strain rates. The values of stress saw an increase of around 12\% for strain rates between 10 and 100/s based on experimental data [3]. Intermediate values are found by interpolating between the curves and if a point falls out of range, the closest curve is utilized. The values for the stressstrain relations at each given rate were found through many rounds of experimental testing [6]. The yield stresses ranged from $52 \mathrm{ksi}(358 \mathrm{MPa})$ for no rate effects to almost $78 \mathrm{ksi}$ (538 MPa) for rates of 100 .

The concrete in both the header and the footer was modeled with the K\&C Concrete Model, *MAT_072 Rcl 3 [7]. The concrete strength was set at $5000 \mathrm{psi}$ (34.5 MPa) and the density at $145 \mathrm{lb} / \mathrm{feet}^{3}\left(60 \mathrm{~kg} / \mathrm{m}^{3}\right)$. To account for the strain rate effects, tabulated data was strain rates ranging from $3.0 \mathrm{e}-4$ to $3.0 \mathrm{c} 4$ were used. The increase factors at each strain rate were determined from calibrated test data provided by $\mathrm{K} \& \mathrm{C}$.

Early on in the simulator development, attempts were made to develop a material model for the urethane programmer [8]. A model was developed [9] to simulate a testing series on concrete masonry walls such that the material could impact the wall through a contact surface applied through the face of the wall and the face of the programmer material. The model chosen was *MAT_057 or *MAT_LOW_DENSITY_FOAM and was selected because of the similarities between the stress-strain behavior and the energy dissipation characteristics between low-density foam and the programmer. The model requires the input of the material density and the material modulus. The unloading is governed by two factors, the HU and the SHAPE. In the LS-DYNA Keyword Manual [5], HU is defined as the hysteretic unloading
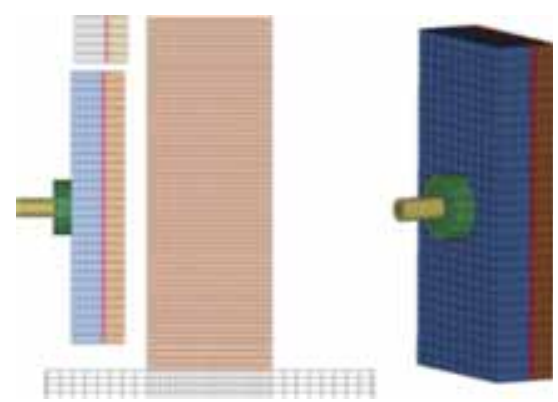

Figure 9: Mesh of impacting module. 
factor between zero and one, where one corresponds to no energy dissipation. SHAPE is defined as the shape factor for unloading. Shape factors greater than one increase energy dissipation and those less than one decrease energy dissipation. The shape of the stress-strain curve (for loading) used by Oesterle [9] is shown in Fig. 10.

The model used in the steel column tests was identical to that used in the modeling of the masonry wall tests, with two slight modifications. The material used to create the column programmer was slightly denser and less stiff than the programmers used for the previous tests. The density was modified from $0.03 \mathrm{lb} / \mathrm{in}^{3}\left(830 \mathrm{~kg} / \mathrm{m}^{3}\right)$ to $0.043 \mathrm{lb} / \mathrm{in}^{3}\left(1,190 \mathrm{~kg} / \mathrm{m}^{3}\right)$ and a scale factor of 2.0 was used to scale the stress-strain curve to make it stiffer than the 1.7 previously used to account for modifications made to the programmer material. The initial modulus was kept the same at $2262 \mathrm{psi}(15.6 \mathrm{MPa})$. The HU of 0.05 and a SHAPE of 400 were also kept consistent.

The model used to represent the rubber connection elements was similar to that used for the programmer. *MAT_LOW_DENSITY_FOAM was used with parameters adjusted to match test data and incorporate the slight rotation in the system. The values of density and modulus were set to 0.043 and 2262, respectively. A HU of 0.05 and a SHAPE of 400 was included.

\subsubsection{Strong axis model results}

An example of the finite element model results from Test SA-10 is shown in Fig. 11 for multiple time steps throughout the run. Additional results for comparison can be found in Tables 2 and 3 of reference [1].

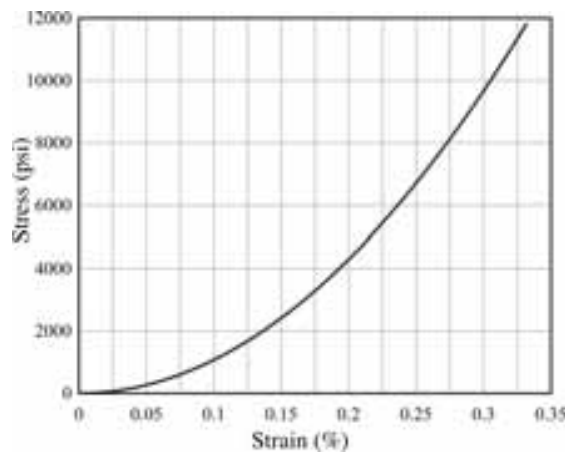

Figure 10: Stress-strain curve for programmer model [8].

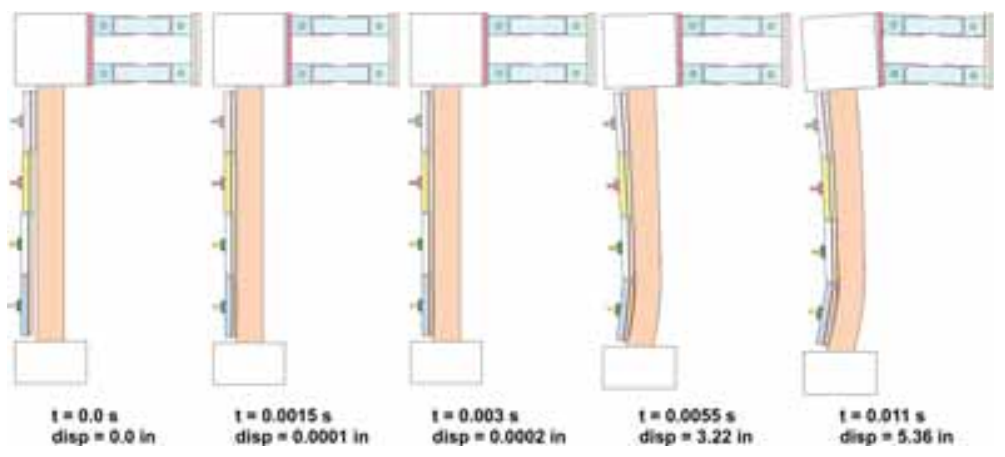

Figure 11: Test SA-10 finite element model. 


\subsection{Weak Axis Loading Model}

The weak axis tests were modeled using the LS-DYNA mesh from the strong axis direction with the column rotated 90 degrees. The model was calibrated with the data from the simulator tests and comparisons are shown in Section 5.2.

\subsubsection{Loading}

The model was identical to that of the strong axis model with the exception of the loading. For the sand experiments, Smooth Particle Hydrodynamics (SPHs) [10] elements were used to model the sand in the inner part of the column as shown in Fig. 12. The sand model used was *MAT_25 or *MAT_GEOLOGICAL_CAP_MODEL. The model was calibrated from U.S. Army Engineer Waterways Experiment Station (WES) flume sand tests by Green [11]. The density of the sand was $0.0614 \mathrm{lb} / \mathrm{in}$. The bulk modulus, the shear modulus and the failure envelope parameter were set to $1.1605 \times 10^{-4} \mathrm{psi}, 0.5915 \times 10^{-4} \mathrm{psi}$ and 0.2238 radians, respectively. The water tests were modeled by applying a varying pressure-time history along the height of the column on both the column web and the inner flanges. These pressure-time histories were computed using the hydrodynamics code, CTH [12].

\subsubsection{Weak axis model results}

The finite element model results from Test WA-06 is shown in Fig. 13 for time steps throughout the run. The figure shows the SPH particles exiting the column area as the bladder is impacted.
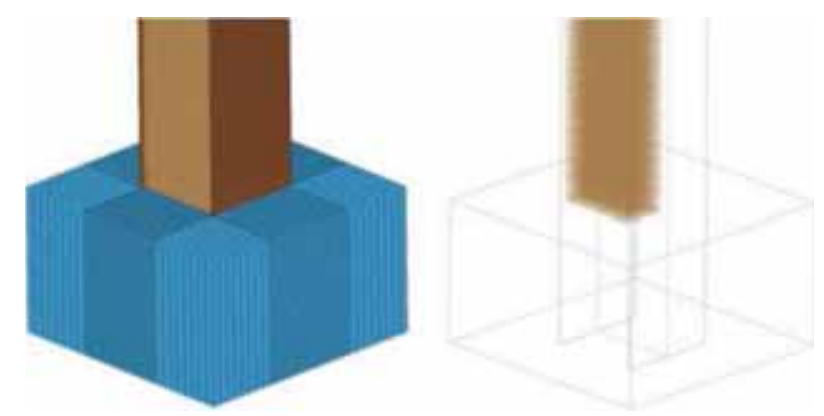

Figure 12: SPH elements for sand modeling.
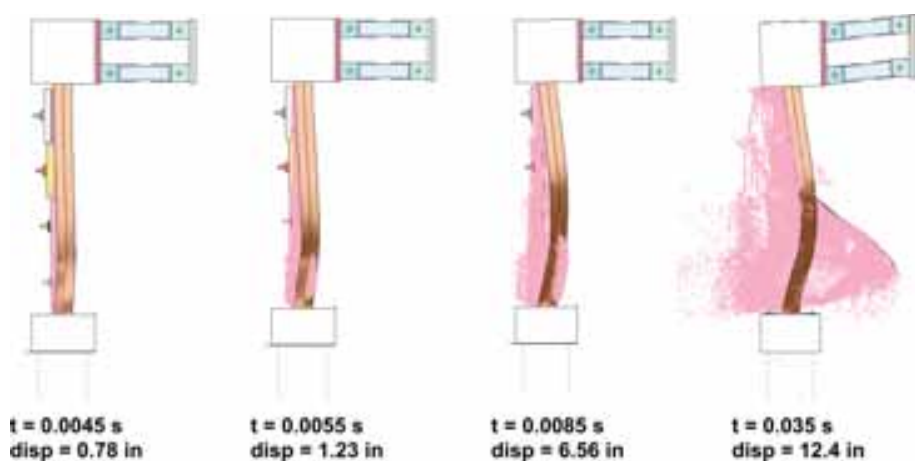

Figure 13: Test WA-06 finite element model results. 


\section{COMPARISONS}

Qualitative and quantitative comparisons were made between the simulated experiments and the computational model for medium and high level tests performed. The results from the experiments against the calibrated model are given in the following sections.

\subsection{Strong axis loading comparisons}

\subsubsection{Test SA-02}

Test SA-02 was a W 10×49 specimen loaded with four BG25s at uniform velocity. From the data analysis, the average of the impact velocity of the BGs was found to be $676.8 \mathrm{in} / \mathrm{s}$ $(17.2 \mathrm{~m} / \mathrm{s})$. The comparisons for the finite element model results and Test SA-02 for both maximum and residual displacements are shown in Fig. 14. The maximum displacement of the finite element model was 1.86 in, and occurred $3.5 \mathrm{~ms}$ after initial contact as compared with a displacement of 1.79 in from the experiment, which corresponds to an error of $3.91 \%$. The residual displacement of the model was 1.10 in and the experimental displacement was 1.09 in. The error from the residual displacements was $0.92 \%$. Included in Fig. 15 is a comparison from the finite element model and the experiment.

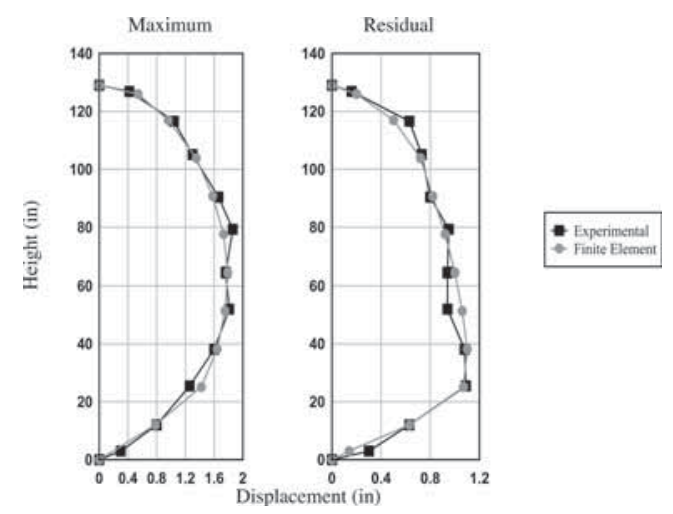

Figure 14: Test SA-02 maximum and residual displacement comparisons.
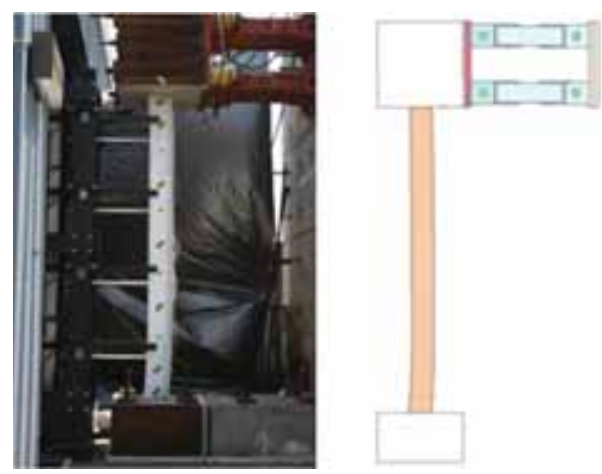

Figure 15: Test SA-02 global posttest comparison. 


\subsubsection{Test SA-03}

Test SA-03 was a W $10 \times 49$ specimen loaded with four BG25s at uniform velocity. From the data analysis, the average of the impact velocity of the BGs was found to be $947.5 \mathrm{in} / \mathrm{s}$ $(24.1 \mathrm{~m} / \mathrm{s})$. The finite element displacement results are plotted against displacements seen in the Test SA-03 experiment in Fig. 16. The maximum displacement of the finite element model was 3.05 in as compared with a displacement of 2.90 in from the experiment, which corresponds to an error of $5.17 \%$. The maximum displacement occurred $4.2 \mathrm{~ms}$ after initial programmer contact. The residual displacement of the computational model was 2.38 in and the experimental displacement was 2.18 in. The error from the residual displacements was found as $9.17 \%$. Included in Fig. 17 are visual comparisons from the finite element calculation and the experiment.

\subsubsection{Test SA-07}

Test SA-07 was a W14×132 specimen loaded at variable velocities. The velocities of the BGs were found to be $1759.4 \mathrm{in} / \mathrm{s}(44.7 \mathrm{~m} / \mathrm{s}), 1456.8 \mathrm{in} / \mathrm{s}(37.0 \mathrm{~m} / \mathrm{s}), 982.0 \mathrm{in} / \mathrm{s}(24.9 \mathrm{~m} / \mathrm{s})$ and $443.5 \mathrm{in} / \mathrm{s}(11.3 \mathrm{~m} / \mathrm{s})$ for BG1 to BG4, respectively. The maximum displacement at a time of $9.8 \mathrm{~ms}$ of the finite element model, shown in Fig. 18, was 4.44 in as compared with a displacement of 4.65 in from the experiment, which corresponds to an error of $4.5 \%$. The residual displacement of the computational model was 3.96 in and the experimental

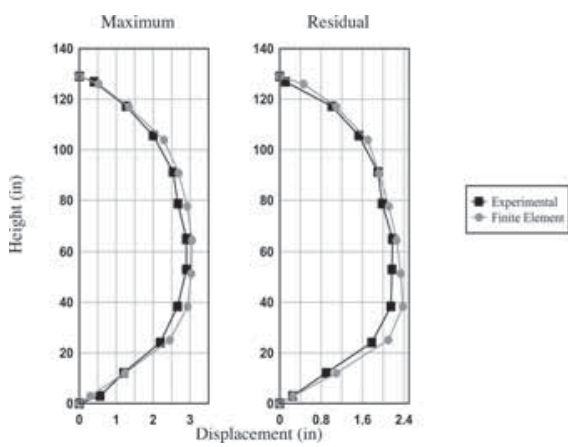

Figure 16: Test SA-03 maximum and residual displacement comparisons.
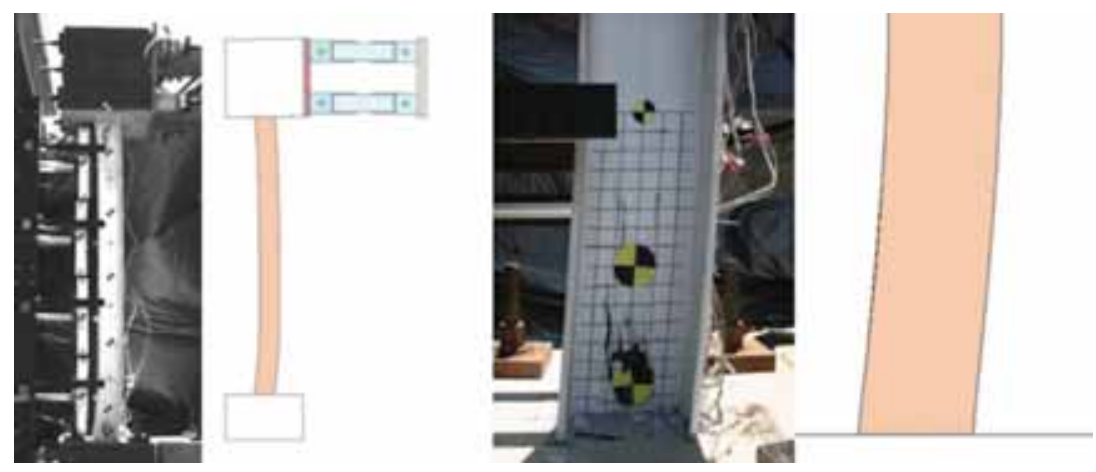

Figure 17: Test SA-03 comparisons of global behavior (left) and base (right). 


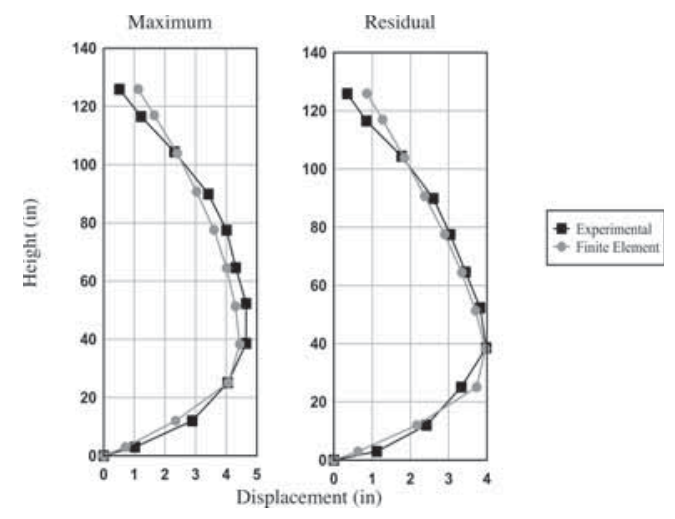

Figure 18: Test SA-07 maximum and residual displacement comparisons.
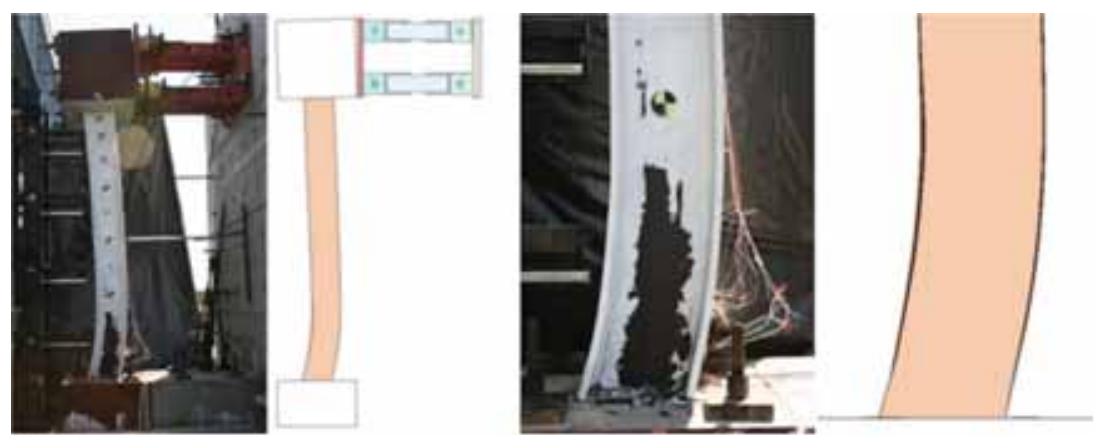

Figure 19: Test SA-07 comparisons of global behavior (left) and base (right).

displacement was $3.98 \mathrm{in}$. The error from the residual displacements was found as $0.05 \%$. Included in Fig. 19 are visual comparisons from the finite element calculation and the experiment.

\subsubsection{Test SA-09}

Test SA-09 was a W14×132 specimen loaded with two BG25s and two BG50s at variable velocities. From the data analysis, the velocities of the BGs were found to be $1780.0 \mathrm{in} / \mathrm{s}$ $(45.2 \mathrm{~m} / \mathrm{s}), 1472.5 \mathrm{in} / \mathrm{s}(37.4 \mathrm{~m} / \mathrm{s}), 987.5 \mathrm{in} / \mathrm{s}(25.1 \mathrm{~m} / \mathrm{s})$ and $442.0 \mathrm{in} / \mathrm{s}(11.2 \mathrm{~m} / \mathrm{s})$ for BG1 to BG4, respectively. The comparisons for the finite element model results and Test SA-09 for both maximum and residual displacements are shown in Fig. 20. The maximum displacement of the finite element model was 4.51 in as compared with a displacement of 4.93 in from the experiment, which corresponds to an error of $5.93 \%$. This occurred $10.4 \mathrm{~ms}$ after the initial programmer contact. The residual displacement of the model was 3.99 in and the experimental displacement was 4.08 in. The error from the residual displacements was $0.23 \%$. Included in Fig. 21 is a comparison from the finite element model and the experiment.

\subsubsection{Test SA-10}

Test SA-10 was a W14×132 specimen loaded at $1759.4 \mathrm{in} / \mathrm{s}(44.7 \mathrm{~m} / \mathrm{s}), 1456.8 \mathrm{in} / \mathrm{s}(37.0 \mathrm{~m} / \mathrm{s})$, $982.0 \mathrm{in} / \mathrm{s}(24.9 \mathrm{~m} / \mathrm{s})$ and $443.5 \mathrm{in} / \mathrm{s}(11.3 \mathrm{~m} / \mathrm{s})$ for BG1 to BG4, respectively. The maximum 


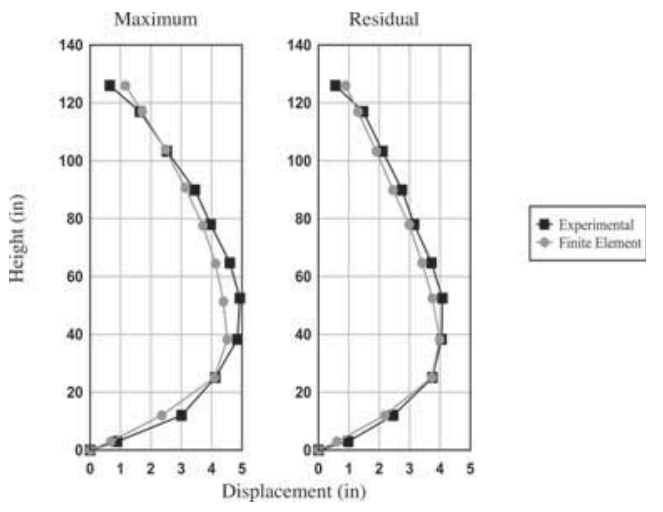

Figure 20: Test SA-09 maximum and residual displacement comparisons.
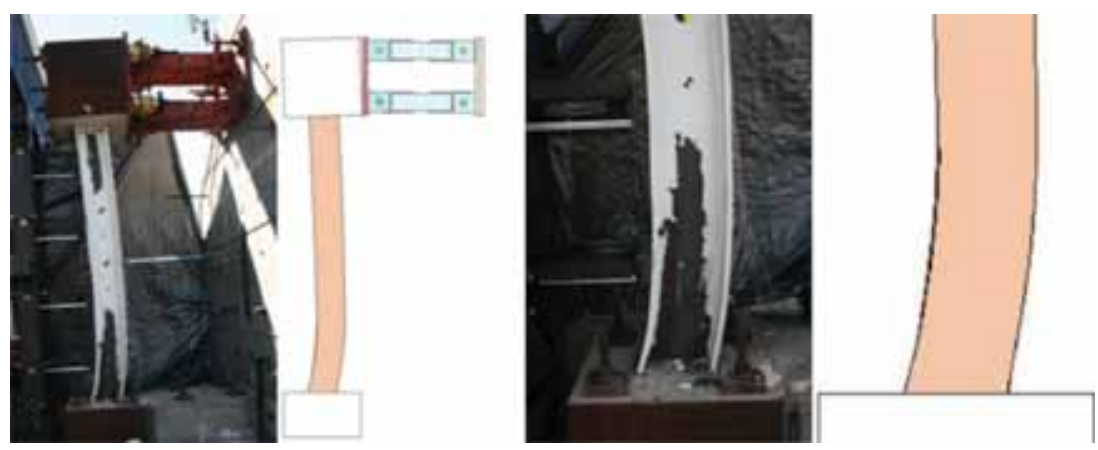

Figure 21: Test SA-09 comparisons of global behavior (left) and base (right).

displacement of the finite element model at a time of $10.0 \mathrm{~ms}$, shown in Fig. 22, was 5.36 in as compared with a displacement of 5.50 in from the experiment, which corresponds to an error of $5.82 \%$. The residual displacement of the computational model was 4.89 in and the experimental displacement was $4.54 \mathrm{in}$. The error from the residual displacements was found as $7.71 \%$. Included in Fig. 23 are visual comparisons from the finite element calculation and the experiment.

\subsubsection{Test SA-11}

Test SA-11 was a W $10 \times 49$ specimen loaded at variable velocities. From the data analysis, the velocities of the BGs were found to be $1594.5 \mathrm{in} / \mathrm{s}(40.5 \mathrm{~m} / \mathrm{s}), 1334.6 \mathrm{in} / \mathrm{s}(33.9 \mathrm{~m} / \mathrm{s})$, $1267.7 \mathrm{in} / \mathrm{s}(32.2 \mathrm{~m} / \mathrm{s})$ and $972.4 \mathrm{in} / \mathrm{s}(24.7 \mathrm{~m} / \mathrm{s})$. Maximum and residual displacements at multiple locations were found along the height of the column. The finite element displacement results are plotted against displacements seen in the Test SA-11 experiment in Fig. 24. From the plots, the maximum displacement of the finite element model was 7.30 in at $11.6 \mathrm{~ms}$ after impact as compared with a displacement of 7.09 in from the experiment, which corresponds to an error of $2.96 \%$. The residual displacement of the computational model was 6.67 in and the experimental displacement was $6.31 \mathrm{in}$. The error from the residual displacements was found as 5.71\%. Included in Fig. 25 are visual comparisons from the finite element calculation and the experiment. 


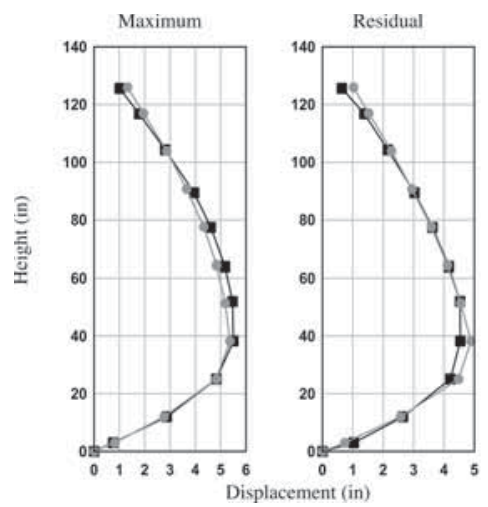

$$
\begin{aligned}
& \text { - Experimental } \\
& \text { - Finite Elenent }
\end{aligned}
$$

Figure 22: Test SA-10 maximum and residual displacement comparisons.
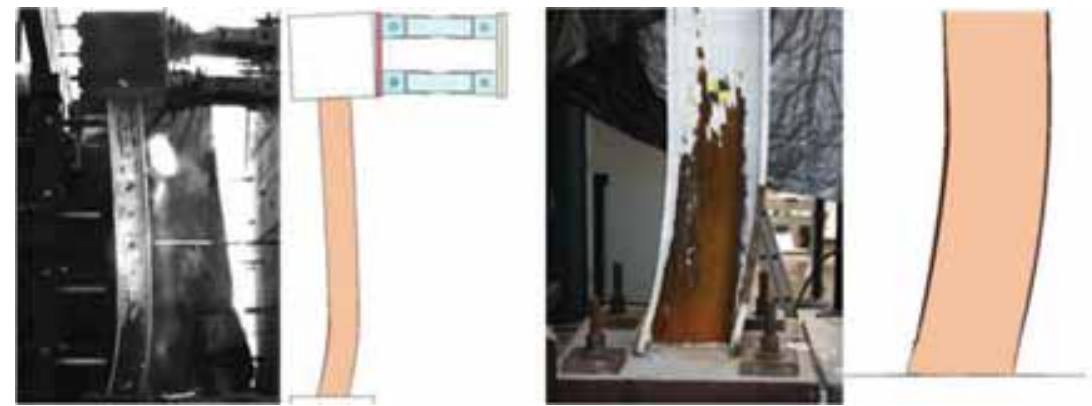

Figure 23: Test SA-10 comparisons of global behavior (left) and base (right).

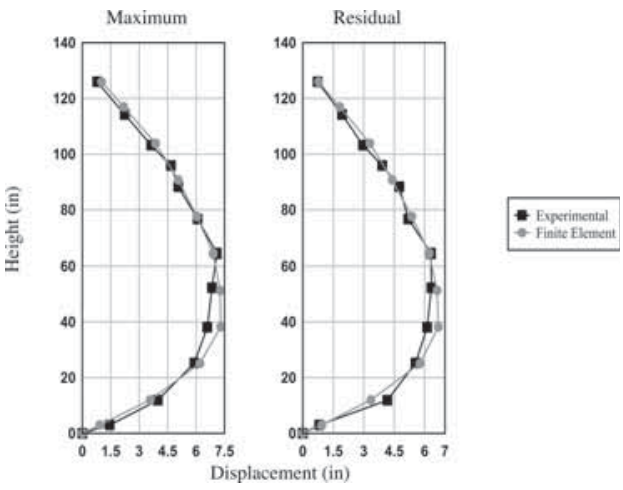

Figure 24: Test SA-11 maximum and residual displacement comparisons. 

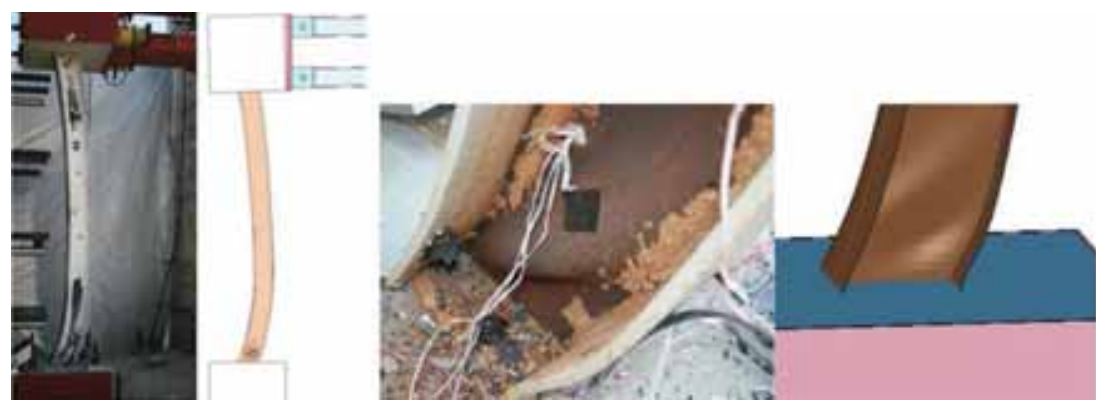

Figure 25: Test SA-11 comparisons of global behavior (left) and base (right).

\subsection{Weak axis loading comparisons}

\subsubsection{Test WA-01}

Test WA-01 was a W10 $\times 49$ specimen loaded at uniform velocity with sand as the loading medium. The average of the impact velocity of the BGs was found to be $676.8 \mathrm{in} / \mathrm{s}(17.2 \mathrm{~m} / \mathrm{s})$. The finite element displacement results are plotted against displacements seen in the experiment in Fig. 26. The maximum displacement of the finite element model at time equal to 23.1 ms was 3.55 in as compared with 3.84 in from the experiment, which corresponds to an error of $8.17 \%$. The residual displacement of the computational model was 2.42 in and the experimental displacement was 2.67 in. The error from the residual displacements was found as 9.36\%. Figure 27 gives visual comparisons from the finite element calculation and the experiment.

\subsubsection{Test WA-04}

Test WA-04 was a W10 $\times 49$ specimen loaded at variable velocity with sand as a loading medium. From the data analysis, the velocities of the BGs were found to be $1759.4 \mathrm{in} / \mathrm{s}(44.6 \mathrm{~m} / \mathrm{s})$, $1456.8 \mathrm{in} / \mathrm{s}(36.9 \mathrm{~m} / \mathrm{s}), 982.0 \mathrm{in} / \mathrm{s}(24.9 \mathrm{~m} / \mathrm{s})$ and $443.5 \mathrm{in} / \mathrm{s}(11.3 \mathrm{~m} / \mathrm{s})$. The finite element displacement results are plotted against displacements seen in the Test WA-04 experiment in Fig. 28. From the plots, the maximum displacement of the finite element model was 4.90 in as compared with a displacement of 5.43 in from the experiment, which corresponds to an error of $9.76 \%$. This occurred at $28.7 \mathrm{~ms}$ after impact. The residual displacement of the computational model was 4.48 in and the experimental displacement was 4.01 in, an error of $11.71 \%$. Figure 29 gives visual comparisons from the calculation and the experiment.

\subsubsection{Test WA-05}

Test WA-05 was a W10×49 specimen with water as a loading medium. Loads were applied to the web and flanges were pressures calculated from CTH. Due to the water obscuring the camera, it was difficult to measure displacements with the camera and therefore only visual comparisons were made and are included in Fig. 30.

\subsubsection{Test WA-06}

Test WA-06 was a W10×49 specimen loaded with sand at variable velocity. The velocities of the BGs were found to be $1780.0 \mathrm{in} / \mathrm{s}(45.2 \mathrm{~m} / \mathrm{s}), 1472.5 \mathrm{in} / \mathrm{s}(37.4 \mathrm{~m} / \mathrm{s}), 987.5 \mathrm{in} / \mathrm{s}(25.1 \mathrm{~m} / \mathrm{s})$ and $442.0 \mathrm{in} / \mathrm{s}(11.2 \mathrm{~m} / \mathrm{s})$. The failure strain of the steel was varied in order to correctly 


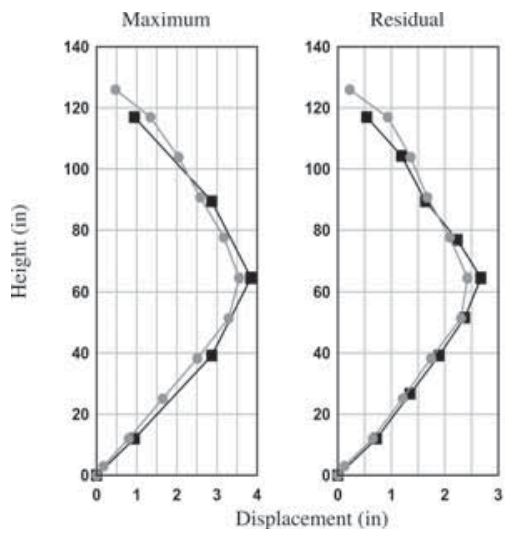

- Experimental

Figure 26: Test WA-01 maximum and residual displacement comparisons.
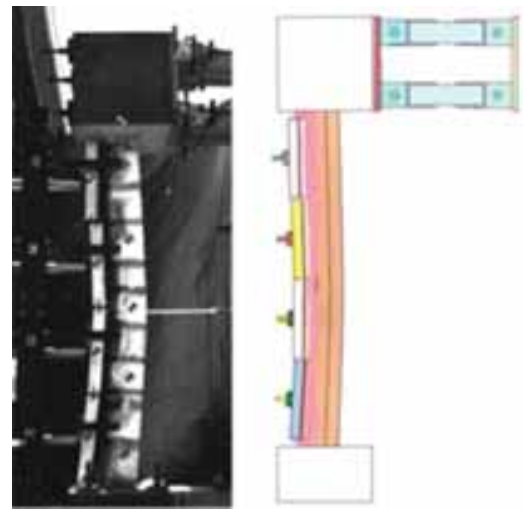

Figure 27: Test WA-01 comparisons of global behavior.

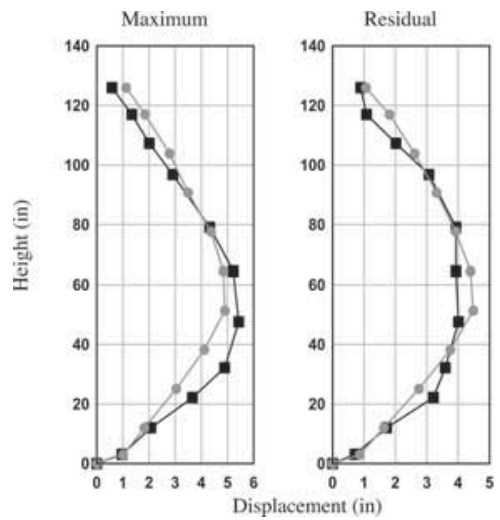

- Experimental
- Finite Flement

Figure 28: Test WA-04 maximum and residual displacement comparisons. 

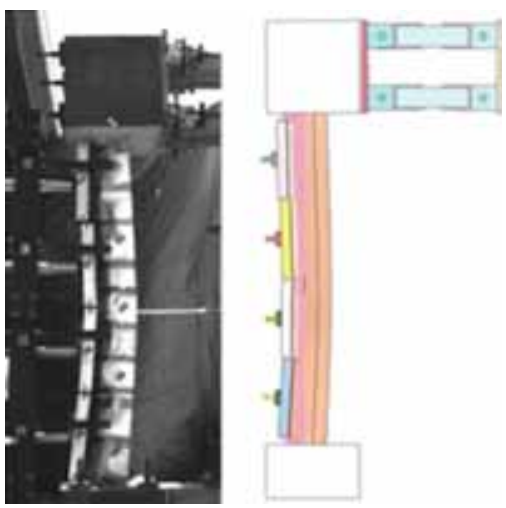

Figure 29: Test WA-04 comparisons of global behavior.
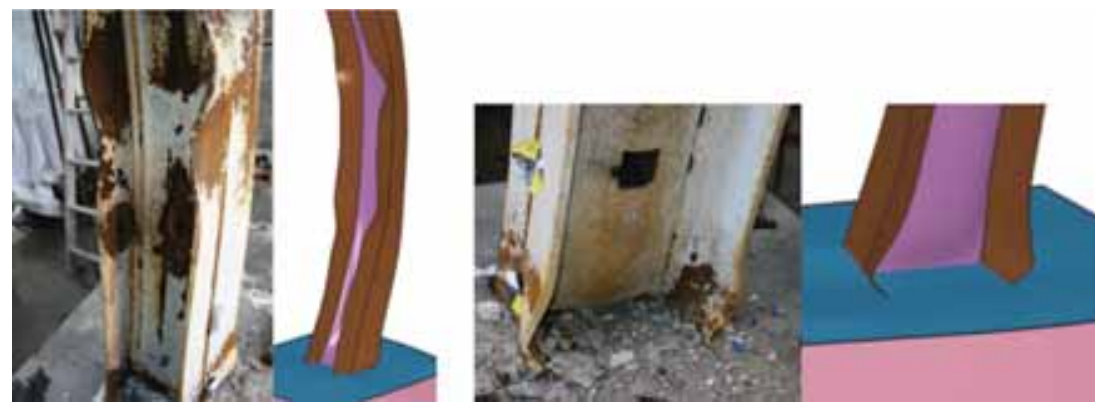

Figure 30: Flange buckling comparisons for Test WA-05.
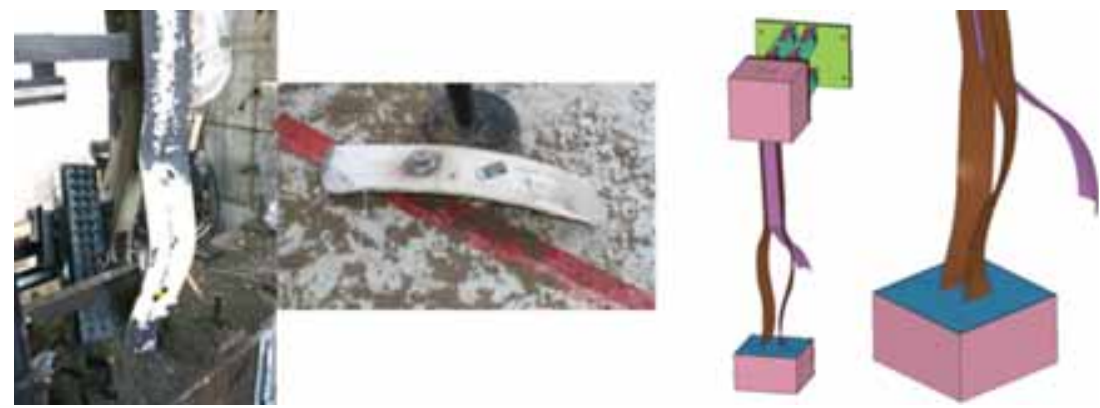

Figure 31: Test WA-06 comparisons of web failure.

capture the flyer plate creation, however, it was not completely successful. Figure 31 gives the results from the current model and shows the web becoming detached, but no flyer plate created. A more detailed model is currently being considered to account for this failure mode.

\section{CONCLUSIONS}

This paper described the procedure for developing and calibrating a finite element model for predicting column response when loaded with the UCSD blast simulator. The steel columns were modeled using shell elements and used a piecewise linear isotropic model that 
incorporated strain rate effects. The use of this model, as well as the correct modeling of the boundary conditions proved to be critical in the overall development of the model. The model can accurately predict the local and global behavior of the column in most tests, including tests that exhibited buckling, however, flyer plate creation from web detachment is not possible. The experiments were compared with the displacements of the model and it was observed that the model did not produce errors for displacements of over $10 \%$.

\section{REFERENCES}

[1] Hegemier, G., Seible, F., Arenett, K., Rodriguez-Nikl, T., Oesterle, M., Wolfson, J., Gram, M. \& Clark, A., The UCSD Blast Simulator. 77th Shock and Vibration Symposium. Monterey, CA, 2006.

[2] Stewart, L., Experimental and computational methods for steel columns subjected to blast loads. WIT Transactions on The Built Environment, Vol. 126, WIT Press: Southampton and Boston, 2012.

[3] Stewart, L., Testing and Analysis of Structural Steel Columns Subjected to Blast Loads, La Jolla, CA, 2010.

[4] Huson, P., Asaro, R., Stewart, L. \& Hegemier, G., Non-explosive methods for simulating blast loading on structures with complex geometry. International Journal of Impact Engineering, 38(7), pp. 546-557, 2011. doi: http://dx.doi.org/10.1016/j.ijimpeng.2010.06.002

[5] Livermore Software Technology Corporation. LS-DYNA Keyword Theory Manual. Livermore, CA, 2007.

[6] Malvar, L., Review of static and dynamic properties of steel reinforcing bars. American Concrete Institute Materials Journal, 95(5), pp. 609-616, 1998.

[7] Malvar, L., Crawford, J., Wesevich, J. \& Simons, D., A plasticity concrete material model of DYNA3S. International Journal of Impact Engineering, 19(9-10), pp. 847-873, 1997. doi: http://dx.doi.org/10.1016/S0734-743X(97)00023-7

[8] Sallay, J. \& Gurtman, G., Characterization of Blast Simulator Programmers, Final Report, Science Applications International Corporation, 2008.

[9] Oesterle, M., Blast Simulator Wall Tests: Experimental Methods and Mitigation Strategies for Reinforced Concrete and Concrete Masonry, La Jolla, CA, 2009.

[10] Randles, P. \& Libersky, L., Smoothed particle hydrodynamics: some recent improvements and applications. Computer Methods in Applied Mechanics and Engineering, 139, pp. 375-408, 1996. doi: http://dx.doi.org/10.1016/S0045-7825(96)01090-0

[11] Green, M., Sheer Friction Test Support Program: Laboratory Test Results for WES Flume Sand Backfill. Vicksburg, MS 1984.

[12] McGlaun, J.M., Thompson, S.L., Kmetyk, L.N. \& Elrick, M.G., CTH: a three dimensional shock wave physics code. International Journal of Impact Engineering, 10(1), pp. 351-360, 1990. doi: http://dx.doi.org/10.1016/0734-743X(90)90071-3 